\title{
VACÍOS EN LA FORMACIÓN INICIAL DE DOCENTES EN MÉXICO. PROGRAMA PARA FOMENTAR EL HABITUS INVESTIGATIVO
}

GAPS IN MEXICO'S INITIAL TEACHER EDUCATION

A PROGRAM TO FOSTER A RESEARCH HABITUS

\section{Volumen 11, Número 3 \\ Setiembre-Diciembre}

pp. 1-24

Este número se publicó el 15 de diciembre de 2011

\author{
Inés Lozano Andrade \\ Jasarmavet Echegaray Bernabé
}

La revista está indexada en los directorios:

LATINDEX, REDALYC, IRESIE, CLASE, DIALNET, DOAJ, E-REVIST@S,

La revista está incluida en los sitios:

REDIE, RINACE, OEI, MAESTROTECA, PREAL, HUASCARAN, CLASCO 


\title{
VACÍOS EN LA FORMACIÓN INICIAL DE DOCENTES EN MÉXICO. PROGRAMA PARA FOMENTAR EL HABITUS INVESTIGATIVO GAPS IN MEXICO'S INITIAL TEACHER EDUCATION A PROGRAM TO FOSTER A RESEARCH HABITUS
}

\author{
Inés Lozano Andrade ${ }^{1}$ \\ Jasarmavet Echegaray Bernabé ${ }^{2}$
}

Resumen: La capacidad de investigación es una competencia indispensable en cualquier persona hoy en día. En el docente, en particular, esta adquiere especial relevancia para la mejora continua de la práctica. Desde la formación inicial, se hace necesario el desarrollo de un habitus investigativo, que debiera promoverse tanto por los profesores como por los alumnos en las escuelas formadoras de docentes. Por lo anterior, este texto es el reporte de una investigación realizada en la Escuela Normal Superior de México, durante el 2010, con la finalidad de formar el habitus investigativo en los futuros docentes. Dicha institución tiene la tarea de formar docentes reflexivos, pero no necesariamente investigadores de su práctica, por lo cual, se creó, ejecutó y evaluó un programa de formación del habitus investigativo. El proceso consistió en retomar los pasos de la investigación reflexiva de la práctica donde se parte de una problematización; luego, se continuó con el diagnóstico fundamentado en un marco teórico. Siguió el programa de intervención y se concluyó con la evaluación de su ejecución. Los resultados mostraron la necesidad de promover, significativamente, la capacidad de investigación como una competencia necesaria para la mejora de la práctica docente.

Palabras clave: FORMACIÓN DOCENTE, FORMACIÓN INICIAL, HABITUS INVESTIGATIVO, INVESTIGACIÓN DE LA PRÁCTICA, MÉXICO

\begin{abstract}
A research skill is an essential competence everyone should have today, but mainly teachers as this helps them improve their teaching practice steadily. Therefore, it is imperative to develop a research habitus during pre-service teacher education in both teacher trainers and teacher students. Based on this argument, this text is a research report which took place during the 2010 school year at Escuela Normal Superior de México (a public Mexican teacher training school), aimed at making a research habitus in future teacher students educated under a reflective teaching model which does not necessarily form researchers of their own practice. Thus, a program to promote a research habitus for teacher students was designed, applied and evaluated. The reflective research of practice process was the posing of a problem, the making of a theoretical framework-based diagnostic, the teacher intervention and its evaluation. These research findings showed the need to foster research skills in future teachers to effectively develop a research competence to improve their teaching practice.
\end{abstract}

Keywords: TEACHER EDUCATION, PRE-SERVICE TEACHER EDUCATION, RESEARCH HABITUS, RESEARCH OF TEACHING PRACTICE, MEXICO

\footnotetext{
Sociólogo, maestro y doctor en Pedagogía de la Universidad Nacional Autónoma de México (UNAM). Ha sido Asesor de UNICEF y miembro del Sistema Nacional de Investigadores. Actualmente profesor investigador de la Escuela Normal Superior de México. Autor de libros y artículos sobre representaciones simbólicas de actores educativos y formación de docentes. Dirección electrónica: iines2101@yahoo.com

${ }^{2}$ Licenciada en Pedagogía, Especialidad en docencia en Educación Normal y Maestra en Docencia y Administración de la Educación Superior. Actualmente profesora en secundaria, catedrática en Escuela Normal Superior de México y en Universidad Católica. Dirección electrónica: j echegaray@live.com.mx
}

Artículo recibido: 4 de setiembre, 2011

Aprobado: 28 de noviembre, 2011 


\section{INTRODUCCIÓN}

En diversos documentos internacionales se ha enfatizado la importancia de la labor del docente en la educación, la sociedad y la economía actual. En su informe a la UNESCO, en el texto denominado "La educación encierra un tesoro", J. Delors afirma que el siglo XXI es visto como una época en la que tanto los individuos como los poderes públicos y privados considerarán la búsqueda de conocimientos como una herramienta esencial, como un medio para el logro de fines, pero, a la vez, como un fin en sí mismo

Esto significa que esperamos mucho del personal docente, que se le exigirá mucho, porque de él depende en gran parte que esta visión se convierta en realidad. El aporte de maestros y profesores es esencial para preparar a los jóvenes. (UNESCO, 1999, p.16)

Lo mismo ocurre con las recomendaciones que la OCDE ha realizado en función del estudio realizado a los docentes y directivos de escuela secundaria en el informe Talis (2009). Derivado del mismo, se hace énfasis en la necesidad de cuidar más los procesos de reclutamiento en las escuelas normales, así como de mejorar los procesos de formación inicial y permanente. Se menciona, además, que la formación del docente es el elemento fundamental para poder mejorar los procesos de aprendizaje en la escuela secundaria.

De esta manera, a nivel nacional e internacional se asume que no sólo la educación se ha convertido en una plataforma para el desarrollo, sino que, dentro de ella, la labor más importante le corresponde al docente y, en particular, a su formación (UNESCO, 1999).

En México, la institución encargada de la formación inicial de profesores es la Escuela Normal. Aunque no se manifiesta explícitamente, su plan actual de estudios (SEP, 1999) se orienta desde el enfoque de la docencia reflexiva, en particular, se abreva en la postura de Schön (1992), quien dice que la reflexión de la práctica en la docencia parte de una crítica a la racionalidad técnico instrumental que se constituyó en el paradigma de formación para todas las profesiones, lo que en palabras del autor significa que se desprofesionalizó al docente al someterlo a una formación tecnocrática que le pedía aplicar, acríticamente, lo que los investigadores educativos habían planteado como medios infalibles para el logro de los propósitos escolares.

El plan de estudios actual, que parte de la crítica a esa racionalidad instrumental, pretende desarrollar diversas competencias en la formación de profesores. Una de ellas 
consiste en que el docente debe de ser lo suficientemente hábil para desarrollar la autocrítica acerca de su práctica, mediante un proceso de reflexión constante que le permita intervenir pertinentemente en ella. Esto implica ser un docente reflexivo de su práctica.

En virtud de que, como se ha señalado infinidad de ocasiones, no se puede enseñar lo que no se tiene, es así como nos proponemos realizar una reflexión de nuestra práctica.

Para ello, hemos adoptado el modelo de investigación de la práctica docente propuesto por Lozano y Mercado (2009), quienes plantean que se debe partir de una problematización de la práctica, seguir con una investigación documental sobre el problema detectado, continuar con un diagnóstico metodológicamente fundamentado, a partir del cual se obtendrá un programa de intervención que se ejecutará y se evaluará después. En este orden de ideas, intentaremos realizar la exposición de este trabajo.

Cabe aclarar que este trabajo se desarrolla en el contexto de la Escuela Normal Superior de México, la cual forma profesores para el nivel secundario y, por lo tanto, existen en esta escuela diferentes especialidades de acuerdo con la asignatura para la cual se está formando al docente. Esto por supuesto tiene implicaciones en cuanto a que la población es heterogénea, pues cada especialidad tiene una subcultura que la caracteriza.

Sin embargo, existen rasgos que las identifican y las conforman tendencias, que nos sirven de base de este documento. El objetivo general de este trabajo es el de fomentar el habitus investigativo en la formación inicial del docente de secundaria para que, de esta manera, se fomenten actitudes y se formen destrezas que se puedan aplicar en la vida cotidiana a mediano y largo plazo en la práctica docente.

\section{Planteamiento del problema}

A partir de nuestra inserción como docentes en las diferentes especialidades de la Licenciatura en Educación Secundaria del plan mencionado de la Escuela Normal Superior de México, se ha podido apreciar una serie de problemáticas que impiden el logro de las competencias deseadas en la formación inicial; pero, también se han identificado lo que se consideran como virtudes de la misma, refiriéndonos con esto a aquellas situaciones que no sólo favorecen el logro de estas competencias, sino que inclusive las superan. Cabe aclarar que este es un esfuerzo de abstracción derivado de nuestra observación continua, donde se encuentran tendencias, pero no generalizaciones, por lo cual es de entender que no solamente las diferentes especialidades y alumnos tienen distintas problemáticas y virtudes. 
Haciendo un recuento de esto, comenzando con las que consideramos virtudes, se presentan las siguientes como un producto de las diversas prácticas de formación inicial:

- El control de grupo, habilidad que es fomentada de diversas maneras dentro de las prácticas normalistas.

- Liderazgo como una capacidad derivada de la anterior y que tiene que ver con la facilidad que tienen los docentes en formación para lograr influencia en los adolescentes.

- Conocimientos psicopedagógicos, y didácticos que aunque dispersos y superfluos, conforman una parte de sus saberes.

- Aprecio por los alumnos de la escuela secundaria.

- Conocimiento y apego a los lineamientos de los planes y programas de estudio.

No obstante, los futuros profesores muestran debilidades en el desarrollo de ciertas competencias para la toma pertinente de decisiones en su quehacer educativo. Al respecto, se hablará de problemas identificados durante el proceso de formación:

- Falta de experiencia, ya que aunque existen prácticas cada vez más frecuentes, aún son insuficientes para poder lograr la inserción adecuada al ambiente escolar al finalizar su carrera.

- Falta de habilidades y conocimientos en la producción de textos escritos.

- Falta de hábito e interés por la lectura.

- Ruptura entre la teoría y la práctica.

- Falta de habilidades para la investigación.

Cierto es que todo lo anterior se promueve poco, pues no conforman una prioridad en el plan de estudios; sin embargo, hay que considerar que la investigación como una competencia que puede ser considerada como aglutinante de todos los anteriores saberes, es básica para cualquier ciudadano de este tiempo y que, además, es indispensable para que el docente pueda ser considerado como un profesional capaz de reflexionar e investigar su práctica, por lo tanto, es un aspecto primordial en la formación inicial. En función de ello es que nos propusimos generar un diagnóstico y programa de intervención acerca de esta problemática. 


\section{Sustento teórico}

Una vez que hemos delimitado la problemática a indagar e intervenir, se hace necesario realizar una recuperación teórica en torno a la misma; para ello, partimos de la necesidad de distinguir entre dos posturas distintas acerca de la reflexión de la práctica docente (Lozano, 2010).

Una que denominaremos empirista, basada en las ideas de Schön (1992) y Perrenoud (2002), quienes enfatizan la labor reflexiva sobre la investigativa, en la queseñalan que no es necesario usar la teoría ni realizar un proceso investigativo en estricto sentido para realizar dicha reflexión y, por su lado, la tendencia con énfasis en la investigación que proponen Stenhouse (1987), Kemmis (1992) y Zeichner (1993) entre otros, quienes parten de la idea de que el docente debe ser un investigador desde y para su práctica, por lo cual enfatizan el empleo de la praxis, es decir, la teoría para explicar y transformar la práctica. Sin pretender hacer aquí una exposición completa del primer enfoque, para los fines que nos interesan realizaremos un esbozo al respecto.

La propuesta de Donald Schön, en torno a la reflexión de la práctica en la docencia, parte de una crítica a la racionalidad técnico instrumental que se constituyó en el paradigma de formación para todas las profesiones desde los dos siglos anteriores, desprofesionalizando al docente al someterlo a una formación tecnocrática que le pide aplicar lo que los investigadores educativos han descubierto como medios infalibles para el logro de los propósitos escolares.

La propuesta de Schön, después de realizar esta crítica a la racionalidad técnica en la formación y práctica de los profesores, pasa por el concepto de profesionalización y especialización derivados de la modernidad y que ya hemos abordado anteriormente como propios de este proyecto.

Para Schön el docente está desprofesionalizado en la medida en que se convierte en un sujeto aplicador de las técnicas descubiertas científicamente. Para profesionalizarlo hace falta considerar de otra manera los saberes que posee. Retomando a Dewey, afirma que la mayoría de los profesionales no aplican los conocimientos científicos en la práctica tal cual los han aprendido en su proceso de formación, sino que en el transcurso de la práctica toman una serie de decisiones que son derivadas de la experiencia, la cual les ha proporcionado un cúmulo de saberes necesarios para ser aplicados en situaciones reales. 
Estas habilidades, destrezas, conocimientos, valores, etc., son considerados los saberes propios del docente que lo hacen o deberían hacerlo un profesional.

Puede decirse que uno de los méritos principales de la teoría consiste en el rescate del docente como un profesional, el cual puede y debe tener un papel activo en la formulación de objetivos, fines de su trabajo, así como en los medios a emplear. Que la enseñanza debe volver a manos de los profesores; que el profesor es capaz también de realizar conocimiento; que ese saber es una teoría, un conocimiento tácito o implícito; que el conocimiento educativo no tiene por qué ser propiedad de unos cuantos, alejados de las aulas en sus posiciones de poder y demeritan, en gran medida, la labor reflexiva e investigativa del docente.

Sin embargo, se ha podido demostrar que en la formación inicial, las prácticas se convierten en un mecanismo de supervisión donde el control del grupo es un parámetro positivo para acreditar las asignaturas. Las planificaciones rigurosas con un exceso de actividades y recursos que difícilmente podrán llevarse a cabo dadas las condiciones de apresuramiento y desinterés por parte de los alumnos en la escuela. La racionalidad técnica continúa su hegemonía, pero disfrazada de un rescate de experiencia a través del diario de campo con algunas narrativas elaboradas a posteriori.

Al respecto, Zeichner dice que en EUA hay cuatro características en la formación de docentes que anulan los intentos emancipadores de esta reforma reflexiva y que, en gran medida, pueden encontrarse también dentro de la formación nacional:

- Un excesivo énfasis en la formación inicial para que los docentes reproduzcan las experiencias de terceros que ya han sido documentadas, o bien, de sus propios profesores en detrimento de la formación de las suyas propias. Lo cual constituye una nueva forma de la racionalidad técnica.

- Un reduccionismo de la reflexión docente a la consideración exclusiva de las destrezas y estrategias docentes necesarias para la enseñanza, obviando aquí los fines de la misma, convirtiendo este proceso en una actividad técnica al servicio de un plan y un programa no diseñado por el docente. Otra forma de la racionalidad técnica.

- Una evidente focalización de los formadores en promover una reflexión de los docentes en formación, centrada en los problemas ocurridos en el aula de manera que se circunscriban a su propia práctica o la de sus alumnos, sin considerar los aspectos sociales que influyen en la enseñanza. La contextualización si acaso se reduce a 
considerar la infraestructura escolar y el personal que la compone, llegando a describirse el clima, flora y fauna de una manera intrascendente para la comprensión de los problemas de aprendizaje.

- La reflexión se individualiza en exceso. Se promueve la idea de que cada maestro tiene sus propios problemas y de que no hay nada que compartir con los demás. Trabajar en solitario se convierte en una idea predominante, aún cuando contradictoriamente, se promueve el colegiado. La comunidad deja de existir. Pensar en los demás es poco usual, muy acorde al discurso neoliberal y posmoderno.

En la docencia reflexiva existen evidencias importantes de que hay un desdén hacia la teoría científica, priorizando casi siempre el saber de la experiencia y la práctica, lo cual evidentemente apuesta a favor del posmodernismo y al neoliberalismo al promoverse el relativismo extremo, que pregona la diversidad de conocimientos y, por lo tanto, la deslegitimación del saber científico.

La visión pragmática y relativista que se encuentra en la práctica de la docencia reflexiva, desvirtúa la esencia de este enfoque que debiera ser profesionalizador y emancipatorio. El énfasis en los relatos individualistas, descontextualizados, subjetivos en gran medida, carentes de una interpretación teórica, que si acaso es extremadamente psicologista y usualmente poco pertinente, sitúa a este enfoque como un elemento que despolitiza la práctica y la formación del docente.

Por lo tanto, respecto a la segunda posición, consideramos con los autores señalados, que ante esta situación una propuesta sencilla emerge: crear condiciones para que los educadores, agrupados en sus espacios de trabajo y acompañados por profesionales que suscriben una visión crítica y ampliada de la ciencia, la investigación y la formación docente, se hagan cargo de sus discursos, miren sus prácticas y muestren sus operaciones, sus logros y caídas a sus estudiantes; que se congreguen en comunidades de investigación de su práctica con fines humanistas y no individualistas; que aprendan a ver su entorno institucional, local, regional, nacional y mundial con una mirada crítica y dejen de pensar en la práctica como si fuera un problema del aula y de medios; que sean más latinoamericanos en su formación y menos occidentales; que vuelvan la mirada a la teoría psicosocio pedagógica para interpretar su realidad y que tengan una formación metodológica para 
indagar su práctica (Messina, 1999; Larrosa, 2007; Lozano y Mercado, 2009; Zeichner, 1993; Lipman, 2001).

El profesor debe reunir información sobre las dimensiones que afectan su entorno educativo y ser capaz de analizar su práctica, organizarla de acuerdo a su importancia, desglosarla y actuar en consecuencia. Para ello, no sólo debe emplear el conocimiento experiencial o de sentido común, pues muchas veces éste revela solo la apariencia de los fenómenos, sino que debe también usar la teoría para trascender el nivel de las apariencias y llegar al de las esencias. Sólo así se podrá tener una comprensión más cabal del fenómeno a fin de poder intervenir en él de manera más fundamentada.

Ubicándonos dentro de ésta última tendencia, es como nos planteamos desarrollar las habilidades investigativas para lograr lo que Perrenoud (2002), ha denominado, retomando a Bourdieu (1996), el habitus para reflexionar e investigar la práctica. Al respecto se define este concepto de la siguiente manera

El habitus es un sistema de disposiciones duraderas adquirido por el individuo en el transcurso del proceso de socialización. Las disposiciones son actitudes, inclinaciones a percibir, sentir, hacer y pensar, interiorizadas por los individuos debido a sus condiciones objetivas de existencia y que funcionan entonces como principios inconscientes de acción, percepción y reflexión. (Bonnewitz, 1998, p. 64)

Determinados esquemas activan nuestros saberes interiorizados, en particular, cuando nos alejamos, analizamos y razonamos. Estos sustentan las interrelaciones, las inferencias, los ajustes a una situación particular, es decir, todas las operaciones de contextualización y de razonamiento sin las que un saber no podría guiar la acción. Es por eso que la formación del habitus es la mediación de saberes y situaciones que exigen una acción.

Una de las funciones de la práctica reflexiva es permitir que el practicante se concientice de sus esquemas. No todas las conductas de los enseñantes son tan automáticas, algunas recurren a los saberes que impulsan un desarrollo, el aprendizaje, la relación, el grupo, etc. Pero el habitus es lo que permite activarlas en la situación; empero, todavía hacen falta esquemas para identificar y aplicar los saberes pertinentes a una situación particular. 
Con respecto a la investigación, hay que mencionar que en el ámbito educativo se ha podido apreciar la importancia de que el docente se vuelva un investigador de su práctica para poderla transformar de manera pertinente y fundamentada.

Diferentes estudios (Ander-Egg, 2005; Carrera, 2000; Talis, 2009) revelan que una de las formas más efectivas para que el docente modifique su práctica, es a través de la investigación de la misma. Al respecto, Latorre (2007, p. 9) menciona que "la enseñanza se concibe como una actividad investigadora y la investigación como una actividad autorreflexiva realizada por el profesorado con la finalidad de mejorar su práctica".

Investigar desde la práctica educativa es una oportunidad para ligar la vida cotidiana con el hacer científico; para usar la experiencia como materia prima del trabajo intelectual y del pensamiento creativo y desarrollar el hábito de reflexionar en y sobre la acción. Permite, además, recuperar las innovaciones pedagógicas, construir saber específico, comprender la acción educativa dentro del contexto social, y comunicar conocimiento emanado del saberhacer.

Con todo lo anterior, y a partir de la revisión de la literatura especializada en este tema, podemos mencionar una serie de actitudes, conocimientos y habilidades que en conjunto harían entre sí lo que llamamos habitus investigativo y que no es más que el conjunto de saberes, disposiciones, habilidades y actitudes interiorizadas y orientadas a la labor constante de investigar reflexivamente la práctica del docente. Para lograr desarrollar este habitus investigativo hemos considerado los siguientes elementos:

- Capacidad para reflexionar la práctica. Lo que implica una actitud constante de autocrítica, alejamiento del sí mismo, apertura hacia los demás y hacia las nuevas ideas (Perrenoud, 2002; Schön, 1992; Manen, Max, 2003).

- Actitud y capacidad de búsqueda y procesamiento de información. Es bien sabido que en las escuelas normales, los alumnos son extremadamente dependientes hacia los profesores y lo que éstos les proporcionen en cuestión de lecturas y textos. Es necesario modificar eso (Perrenoud, 2002; Lozano y Mercado, 2009).

- Habilidad para vincular la teoría y práctica. Aparte del desdén que existe por muchos de los profesores formados y en formación hacia la teoría psicosociopedagógica y probablemente como consecuencia de lo mismo, existen serias dificultades para realizar articulaciones de lo que se vive en la práctica docente con los diferentes 
documentos y autores conocidos (Latorre, 2003; Zeichner, 1993; Lozano y Mercado, 2009).

- Conocimiento de métodos y técnicas de investigación de campo y su utilización adecuada y pertinente. Ya que la formación inicial no implica formar investigadores de acuerdo al plan de estudios reciente, este saber es poco buscado y promovido por los docentes de las instituciones normales. Sin embargo, como hemos visto, es un saber fundamental para poder desarrollar cambios en la práctica. Lo cual seguramente repercutiría en una formación permanente si se logra desarrollar el habitus (Latorre, 2003; Zeichner, 1993; Lozano y Mercado, 2009).

\section{Diagnóstico}

\subsection{Esbozo Metodológico}

Para la realización del diagnóstico, se emplearon técnicas de recopilación de información y de análisis de corte cualitativo con la finalidad de describir e interpretar las prácticas, saberes, habilidades y actitudes investigativas de los alumnos de la Escuela Normal Superior de México, en específico, de un grupo del segundo semestre de la especialidad en pedagogía. Este grupo fue seleccionado por encontrarse al inicio de su proceso de formación, además de que mostró disposición para la labor a realizar una vez que se les explicó la misma.

Las técnicas mencionadas fueron la entrevista semiestructurada y estructurada en las que se indagó respecto a los saberes fundamentales de investigación que tenían los alumnos que hayan aprendido previamente en el nivel bachillerato. En las que se incluyeron cuestiones sobre su conceptualización y experiencia en investigación, encaminadas a una autoevaluación para verificar de manera crítica sus propios conocimientos, además de corroborar que en su experiencia como estudiantes han llevado a cabo trabajos para desarrollar esta competencia investigativa, finalmente que de forma proactiva se propongan nuevas formas de incluir la investigación en las habilidades básicas de los docentes considerándola una manera de profesionalizar la práctica, es así que se incluyeron preguntas como: ¿Qué es para ti investigación? De los trabajos realizados en tu experiencia educativa ¿Cuáles son investigativos? ¿Qué importancia le atribuyes a la investigación en tu formación como educador? ¿ldentificas diferentes técnicas de recopilación de información? 
¿Cuáles? ¿De qué manera analizas y procesas información? ¿De dónde obtienes información cuando haces alguna consulta? Si es internet ¿Qué páginas consultas?, etc.

Estas técnicas fueron acompañadas de una escala de valoración de corte cuantitativo con el fin de complementar la información y lograr el propósito mencionado. En la que se hicieron afirmaciones con cuatro posibilidades de dominio por parte de los alumnos, con la finalidad de saber hasta qué punto los alumnos consideran que han aplicado y desarrollado habilidades investigativas básicas a lo largo de su vida académica (Lo domino, Casi lo domino, Algunas veces lo domino, Me cuesta trabajo, No lo sé hacer).

Algunas afirmaciones como:

- Expresas con facilidad de forma oral y escrita el contenido de un texto.

- Elaboras revisión bibliográfica actualizada y estudios similares en diferentes fuentes.

- Conoces las técnicas de la investigación documental.

- Conoces los elementos fundamentales que debe llevar una referencia bibliográfica.

- Identificas y conoces tendencias teóricas pedagógicas, psicológicas y sociológicas que sustentan el proceso educativo.

- Redactas informes o escritos como un ensayo con sustento y argumentación.

La información que se rescató de estos instrumentos se procesó de manera cualitativa, tratando de encontrar las tendencias más significativas, siempre auxiliándonos de procedimientos estadísticos, pero sin dejar de buscar la descripción y la comprensión que los sujetos otorgan al acto de poseer el habitus investigativo como un elemento fundamental para el desarrollo de sus prácticas docentes.

\subsection{Los resultados del diagnóstico}

Los alumnos de segundo semestre de Pedagogía tienen como estado civil la soltería, en su gran mayoría mujeres, con una formación previa en bachilleratos generales sobre todo del gobierno del Estado de México, ya que la mayoría de estos alumnos y alumnas provienen de municipios de esta entidad. Cuentan con amplias expectativas respecto a la carrera, disposición para el trabajo. Aunque hasta ese momento, se caracterizaba por ser un grupo con participación oral escasa, pero no escrita. 
Con respecto a las categorías buscadas para conocer el estado del habitus investigativo en estos alumnos, sin abundar en evidencias encontradas a través del uso de los instrumentos, se encontró de manera sintética lo siguiente:

\subsubsection{Reflexión de la práctica}

Los alumnos no dominaban la expresión escrita en la toma de apuntes de sus diferentes clases, considerando que tomar apuntes es una habilidad que apoya el aprendizaje al mantener activo al alumno mientras escucha, además, sirve de memoria externa a la cual se puede recurrir para repasar y consultar.

Los alumnos consideran que investigar es sólo lo referente a la búsqueda de información en diferentes fuentes; sin embargo, no cuentan con los elementos para llevar a cabo una investigación documental como lo refirieron, además de realizarla sólo por exigencia de sus profesores y no como una oportunidad de aprendizaje:

"considero que busco la información de distintas fuentes y luego los datos recabados trato de interpretarlos y lo que encuentro me es útil".

"al realizar una exposición porque tengo que investigar y llegar a conclusiones, resolver cuestionarios, análisis, trabajo de perspectivas en dibujo porque el maestro no enseñó el tema y tuve que preguntar a amigos, revisar libros, ver en internet las técnicas y emplearlas para terminar mi trabajo".

\subsubsection{Búsqueda y procesamiento de información}

Los alumnos no reconocen las formas en que procesan información documental aunque las han aplicado en su vida académica por exigencias de los docentes; no obstante, les cuesta trabajo identificarlas y aplicarlas con eficacia para facilitar su aprendizaje autónomo. Asimismo, desconocen fuentes especializadas para hacer consultas y las que hacen son en bibliotecas generales o en páginas de la internet con información muy elemental, la mayoría de los alumnos mencionaron hacer una relación de la teoría con la realidad, pero no escribieron de qué forma lo llevan a cabo, que precisamente sería la forma en que procesan la información, al respecto dicen: 
"articulando todos los textos que he leído y confrontando la teoría con lo visto en las prácticas de observación".

"de la biblioteca (escuela, hogar) internet (google, wikipedia) reviso las fuentes de donde proviene la información y la verifico".

\subsubsection{Vinculación de la teoría y práctica}

Los alumnos encuentran poco significado en la teoría que revisan en la escuela Normal, hallan poca relación de ésta con sus observaciones en la escuela secundaria; no emplean las diferentes teorías que revisan durante su formación para sustentar argumentos $\mathrm{u}$ opiniones al respecto del proceso educativo y desconocen conceptos necesarios para el desarrollo de su profesión docente.

Con relación a esto, se incluyó una pregunta referente al papel que juega la investigación educativa en relación con los paradigmas epistemológicos y el desarrollo de la ciencia y la tecnología en este nuevo siglo, y la mayoría respondió no saber a lo que se refería la cuestión, cuando son contenidos que revisan paralelamente a este curso, a través de diferentes autores y asignaturas entre ellas: Epistemología y paradigmas de la investigación educativa y Sociología de la educación.

\subsubsection{Metodología de la investigación}

Los alumnos consideran que la investigación se reduce al aspecto documental, no cuentan con elementos para llevarla a cabo de forma adecuada, sus consultas son de fuentes poco confiables aun contando con los recursos tecnológicos. No saben las diferencias entre el método cualitativo y cuantitativo ni su aplicación en la investigación. No identifican las diferencias entre una técnica y un instrumento de investigación ni su pertinencia de acuerdo con la naturaleza de la investigación a realizar. Respecto a estos puntos, los alumnos responden lo siguiente:

"investigar es responder a una incógnita con sustentos teóricos sabiendo revisar diversas fuentes y seleccionando información útil".

"investigo en la biblioteca escolar, libros en mi casa, de internet no consulto páginas específicas, me baso en la información que se emplea". 
"lo entiendo como en una entrevista, recabar datos. cuantitativos: edad, peso, talla. Cualitativos: estado civil, sexo, dirección".

"solo porque mi maestra lo pide, para analizar los comportamientos y aprendizajes de los adolescentes con el diario y las entrevistas".

Para iniciar la propuesta era necesario este diagnóstico que permitiera identificar necesidades y de esta forma plantear las acciones pertinentes para satisfacerlas, mismas que a continuación se mencionan:

1. Desarrollo de la expresión escrita para apoyar a la expresión oral.

4. Identificación de las diversas formas de procesamiento de información documental y de campo.

5. Vincular las diferentes teorías con las experiencias en escuela secundaria para argumentar sus intervenciones orales y escritas.

6. Identificación de los métodos de investigación para su adecuado empleo.

7. Identificación de técnicas e instrumentos de investigación para su uso según la naturaleza de la investigación.

8. Conocimiento de diferentes fuentes especializadas para llevar a cabo una selección de información útil para sus investigaciones.

9. Conocimiento y uso de conceptos especializados para su quehacer docente.

\section{Intervención Educativa}

Los espacios curriculares de Estrategias para el Estudio y la Comunicación I y II de la Licenciatura en Educación Secundaria, de acuerdo con el Plan de estudios para las Escuelas Normales de 1999, tienen como finalidad propiciar en los alumnos de la Licenciatura en Educación Secundaria el fortalecimiento de las competencias de lectura comprensiva y crítica, así como la expresión clara en forma oral y escrita, componentes fundamentales del trabajo intelectual que los estudiantes requieren en sus actividades formativas y en su futuro como educadores.

El programa agrupa las actividades de estudio en cuatro campos que permiten sistematizar la formación gradual de las habilidades intelectuales y orientar la selección de los temas de trabajo. Estos campos están presentes a manera de ejes temáticos que se atienden simultáneamente a lo largo de los dos cursos: I) El aprovechamiento de la 
información transmitida oralmente. II) La expresión oral fluida y coherente. III) La lectura de libros y el manejo de las fuentes de información. IV) La redacción de textos y reportes académicos breves.

Es así que la intervención educativa se desarrolló en el contexto de estas dos asignaturas curriculares denominando al programa de la siguiente manera: "EL DESARROLLO DEL HABITUS INVESTIGATIVO EN LOS ESTUDIANTES DE SEGUNDO SEMESTRE DE PEDAGOGÍA".

Para la intervención con los alumnos, se llevó a cabo un taller denominado "Cómo desarrollar el habitus investigativo", cuyo propósito fue: Desarrollar en el alumno normalista de segundo semestre de la especialidad en pedagogía el habitus investigativo, que implica una serie de habilidades como la expresión oral y escrita, la selección, comprensión, jerarquización, procesamiento y sistematización de información para llevar a cabo investigaciones críticas, fundamentadas y propositivas con la finalidad de mejorar la práctica educativa.

Se eligió el taller porque es una situación privilegiada de aprendizaje comunitario con el propósito principal de reflexionar sistemáticamente sobre conocimientos, valores, actitudes y prácticas en cuanto a determinada problemática y que tiene importancia en la vida diaria de cada participante. Como podemos apreciar en la siguiente carta descriptiva del programa, partimos de las necesidades detectadas, aun cuando esto aparezca en la última columna sólo con fines expositivos, de ahí se plantean los propósitos a lograr, de los cual se derivan los elementos didácticos comunes: actividades, recursos, responsables y evaluación. 
Carta descriptiva del programa de intervención

\begin{tabular}{|c|c|c|c|c|c|}
\hline PROPÓSITOS & ACTIVIDADES & RECURSOS & RESPONSABLE & EVALUACIÓN & $\begin{array}{l}\text { NECESIDAD } \\
\text { DETECTADA }\end{array}$ \\
\hline $\begin{array}{lr}\text { Sistematizar } & \text { y } \\
\text { aprovechar } & \\
\text { información } & \\
\text { transmitida } & \text { de } \\
\text { forma oral y } & y \\
\text { escrita. } & \end{array}$ & $\begin{array}{lr}\text { Elaboración de } & \text { de } \\
\text { narraciones de } \\
\text { sucesos importantes } \\
\text { de ros } \\
\text { observaciones en } \\
\text { escuela secundaria } \\
\text { para identificar } \\
\text { aspectos de forma y } \\
\text { fondo en ra } \\
\text { redacción como } \\
\text { congruencia, } \\
\text { coherencia, } \\
\text { ortografía, } \\
\text { puntuación, } \\
\text { separación de } \\
\text { párrafos, el uso de } \\
\text { descripciones dentro } \\
\text { de ésta. } \\
\text { Elaboración de toma } \\
\text { de apuntes en } \\
\text { conferencias sobre } \\
\text { investigaciones } \\
\text { educativas. }\end{array}$ & $\begin{array}{l}\text { Diario de } \\
\text { campo. }\end{array}$ & Profesores & Rúbricas & $\begin{array}{l}\text { Desarrollo de } \\
\text { la expresión } \\
\text { escrita para } \\
\text { apoyar a la } \\
\text { expresión oral. } \\
\\
\\
\text { Relacionar las } \\
\text { diferentes } \\
\text { teorías con las } \\
\text { experiencias } \\
\text { en escuela } \\
\text { secundaria } \\
\text { para } \\
\text { argumentar sus } \\
\text { intervenciones } \\
\text { orales y } \\
\text { escritas. }\end{array}$ \\
\hline $\begin{array}{l}\text { Expresar de } \\
\text { forma oral, fluida } \\
\text { y coherente sus } \\
\text { ideas } \\
\text { argumentos } \\
\text { sólidos. }\end{array}$ & $\begin{array}{l}\text { Realizar lecturas } \\
\text { especializadas, para } \\
\text { esquematizar la } \\
\text { información y poseer } \\
\text { argumentos para } \\
\text { expresarlos de forma } \\
\text { oral. } \\
\text { Exposición oral de } \\
\text { textos } \\
\text { especializados en } \\
\text { educación, } \\
\text { buscando relaciones } \\
\text { con la realidad de la } \\
\text { escuela secundaria. }\end{array}$ & $\begin{array}{l}\text { Textos } \\
\text { especializados }\end{array}$ & Profesores & Rúbricas & $\begin{array}{l}\text { Identificación } \\
\text { de las diversas } \\
\text { formas de } \\
\text { procesamiento } \\
\text { de información. } \\
\text { Conocimiento } \\
\text { de términos } \\
\text { necesarios en } \\
\text { su quehacer } \\
\text { docente. }\end{array}$ \\
\hline $\begin{array}{l}\text { Emplear } \\
\text { diversas fuentes } \\
\text { de información } \\
\text { en sus sus } \\
\text { investigaciones, } \\
\text { para la } \\
\text { elaboración de } \\
\text { reportes } \\
\text { académicos } \\
\text { breves. }\end{array}$ & $\begin{array}{l}\text { Revisión } \\
\text { bibliográfica } \\
\text { actualizada para la } \\
\text { elaboración de } \\
\text { reportes académicos } \\
\text { breves (investigación } \\
\text { en diversos textos, la } \\
\text { internet, revistas } \\
\text { especializadas). de } \\
\text { Búsqueda soda de } \\
\text { información sobre } \\
\text { alguna temática en } \\
\text { bancos electrónicos } \\
\text { de información sobre } \\
\text { investigación } \\
\text { educativa. }\end{array}$ & $\begin{array}{l}\text { Revistas } \\
\text { especializada, } \\
\text { la internet. } \\
\text { Aula de } \\
\text { medios }\end{array}$ & Profesores & Rúbricas & $\begin{array}{l}\text { Conocimiento } \\
\text { de diferentes } \\
\text { fuentes } \\
\text { especializadas } \\
\text { para llevar a } \\
\text { cabo una } \\
\text { selección de } \\
\text { información útil } \\
\text { para sus } \\
\text { investigaciones }\end{array}$ \\
\hline
\end{tabular}




\begin{tabular}{|c|c|c|c|c|c|}
\hline $\begin{array}{lr}\text { Conocer } & \text { la } \\
\text { metodología de } \\
\text { investigación } \\
\text { que se puede } \\
\text { emplear en } \\
\text { educación. }\end{array}$ & 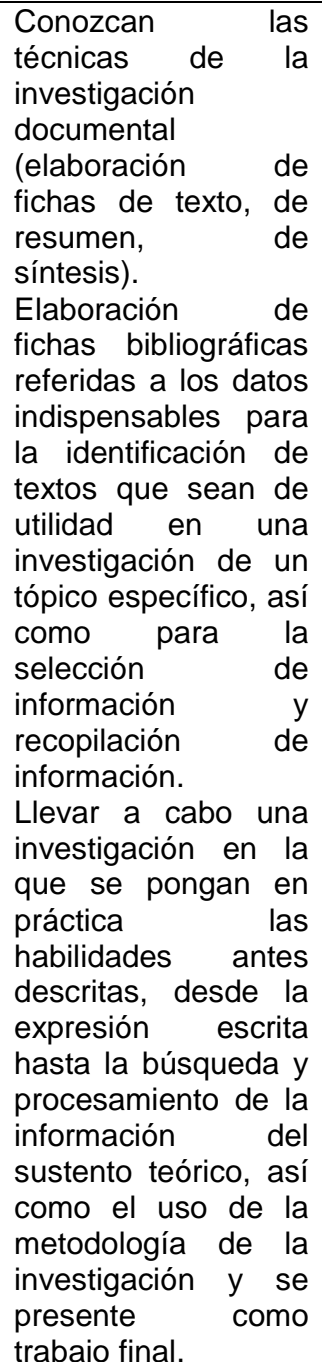 & $\begin{array}{l}\text { Textos de } \\
\text { métodos y } \\
\text { técnicas de } \\
\text { investigación } \\
\text { Fichas de } \\
\text { trabajo. } \\
\text { Fichas } \\
\text { bibliográficas. } \\
\text { Textos } \\
\text { especializados }\end{array}$ & Profesores & $\begin{array}{l}\text { Rúbricas } \\
\text { Autoevaluación }\end{array}$ & $\begin{array}{l}\text { Identificación } \\
\text { de los métodos } \\
\text { de } \\
\text { investigación } \\
\text { para su } \\
\text { adecuado } \\
\text { empleo. } \\
\text { Identificación } \\
\text { de técnicas e } \\
\text { instrumentos } \\
\text { de } \\
\text { investigación } \\
\text { para su uso } \\
\text { según la } \\
\text { naturaleza de } \\
\text { la } \\
\text { investigación. }\end{array}$ \\
\hline
\end{tabular}

\section{Fundamentación teórica del programa}

Una de las teorías desde la que se operó esta propuesta de intervención es la tendencia constructivista con la intención de que los alumnos desarrollen el habitus investigativo a través del aprendizaje significativo por recepción, Ausubel (1976), por descubrimiento, Piaget (1964) y enseñanza de expertos a novatos, Vigotsky (1979).

El enfoque pedagógico constructivista es el más adecuado para la aplicación de los principios filosóficos de la educación para el futuro, crea zonas de desarrollo próximo y reta al alumno a recorrerlas (Vigotsky, 1979). Ayuda al proceso de construcción del alumno, desde las experiencias, intereses y conocimientos previos. El profesor facilita el ambiente, para que el alumno sea el protagonista de su proceso de aprendizaje, sobre un objeto de la 
realidad o contenido de aprendizaje para el desarrollo cognoscitivo, la adquisición de habilidades, actitudes y valores.

El aprendizaje no es el resultado de la copia o repetición de la realidad, sino de un proceso dinámico e interactivo con la misma mediante el cual la información es interpretada y reinterpretada en la mente que construye a la vez, modelos explicativos cada vez más complejos. Este aprendizaje es a decir de Díaz Barriga, significativo: "Durante el aprendizaje significativo el alumno relaciona de manera no arbitraria y sustancial la nueva información con los conocimientos y experiencias previas y familiares que posee en su estructura de conocimientos o cognoscitiva" (1993, p. 33).

Otra de las teorías en que se fundamenta la propuesta es la pedagogía crítica que reconoce el carácter de clase de la educación, cuya praxis es aquella que transforma la realidad, y una de las formas de conseguir ésta es mediante la investigación en el ámbito pedagógico, por lo tanto, en la enseñanza y el aprendizaje. Las ventajas que ésta nos muestra es "el sometimiento a la prueba de la práctica de las ideas como medio para mejorar y de lograr el medio del conocimiento acerca de los planes de estudio, la enseñanza y el aprendizaje" (Lozano, 2006, p. 88), es decir, el resultado es una mejora en aquello que ocurre en las clases y escuela, además de una articulación y justificación mejores de la argumentación educacional aplicada a aquello que sucede.

La propuesta que se presenta se elaboró considerando algunas de estas premisas desde la elaboración del diagnóstico que permitiera dar cuenta de los conocimientos previos para planificar las diversas actividades durante la intervención. Al realizar todas las actividades de la intervención, se pretendió permitir a los alumnos su constante participación en las actividades, manifestación de dudas, crítica constructiva al trabajo llevado a cabo tanto por ellos como por los coordinadores. Esto con la finalidad de hacer sugerencias de mejora, respecto a la forma de conducción como en las mismas actividades propuestas que a continuación de detallarán.

\section{Evaluación del programa de intervención}

En este apartado se describen las actividades que se realizaron, el proceso que se siguió en su desarrollo y el impacto que tuvo en los alumnos. Para realizar esta evaluación, se recurrió a la observación, una rúbrica y entrevistas a los participantes. Esta evaluación se realiza con base en los objetivos planteados en el programa de intervención. Cabe decir que 
estos objetivos se encuentran transversalmente trabajados en las diversas actividades realizadas, es decir, cada actividad tocaba uno o más de estos por lo cual se procede a explicar la forma en que se desarrollaron las actividades y dentro de ellas los objetivos que fueron o no lográndose.

Las actividades se llevaron a cabo durante todo el semestre. Iniciando con las narraciones hechas por los alumnos de forma escrita en su diario de campo y que, además, emplearon para su análisis en otras asignaturas. La toma de apuntes la realizaron al ser espectadores de conferencias con la finalidad de hablar acerca temas de educación básica, éstas les resultaron significativas, porque los temas eran de su interés, además de acercarlos a trabajos reales de investigación. La expresión oral se llevó a cabo en clase con la revisión y sistematización previa de textos especializados que les proporcionaran argumento para ello.

Estas actividades se evaluaron con la rúbrica correspondiente a cada una en las que se consideraron aspectos como congruencia, claridad en las ideas, ortografía, identificación de ideas centrales del tema, seguridad al hablar, etc. Los alumnos manifestaron un gran avance para dar argumento a sus críticas y reportes entregados al incluir referentes teóricos en sus discursos de forma oral y escrita.

La siguiente actividad fue que los alumnos hicieran búsqueda de información en fuentes más confiables y actuales, se revisaron artículos en revistas electrónicas como IRESIE y DIALNET (Bancos electrónicos de información sobre investigación educativa) lo cual dio oportunidad a los alumnos a adentrarse en temas con mayor profundidad y con carácter científico, esto les aportó elementos no sólo para la realización de reportes académicos de la asignatura, sino para otras también. Se evaluó con la rúbrica que consideró aspectos como argumentación, congruencia, claridad en las ideas, puntuación y ortografía.

Las últimas actividades estuvieron muy relacionadas, puesto que se refirieron al uso y aplicación de metodología de la investigación en educación y llevar a cabo precisamente un trabajo final de carácter investigativo como grupo, en el que pusieran en práctica todo lo aprendido en el taller, desde la redacción, ortografía, puntuación, hasta la interpretación de información teórica y empírica con sustento para la argumentación en todo el trabajo.

Comenzaron con la sistematización de información, desde su selección y luego su jerarquización como sustento teórico para la problemática por ellos seleccionada: ¿Qué, 
cuándo y cómo hacen investigación los alumnos normalistas? Para lo que hubo necesidad de una participación ordenada para el establecimiento de categorías conceptuales a indagar; luego, la selección método y técnicas de investigación de campo, así como la elaboración de instrumentos de recogida de información acordes al tipo de estudio, misma que realizaron satisfactoriamente. Posteriormente, interpretaron lo que obtuvieron con su aplicación (con compañeros de otros semestres y especialidades de la Normal), pues al elegir como instrumento el cuestionario y la encuesta se vieron en la necesidad de sistematizar también esta información y con ello de herramientas estadísticas para analizarla e interpretarla, incluyendo el uso triangulado y pertinente de citas de diversos autores para un argumento más sólido.

Para evaluar estas actividades se consideraron los productos obtenidos, es decir, el trabajo de investigación por escrito, su presentación por algunos representantes del grupo que incluyera los elementos necesarios como: planteamiento del problema, análisis del sustento teórico en fuentes de consulta confiable y especializada en la temática, procesamiento de información adecuado, descripción de método, técnicas e instrumentos empleados en los que se identificó el nivel de estructuración de los mismos de acuerdo con los propósitos, sistematización de información, es decir, la forma en la que analizaron e interpretaron la información teórica y empírica, y finalmente el nivel de reflexión de ésta para llegar a sus propias conclusiones.

Puesto que el propósito de la intervención fue desarrollar en estos alumnos normalistas el habitus investigativo, lo cual implico la aplicación de habilidades específicas como expresión oral y escrita, selección, comprensión, jerarquización, procesamiento, sistematización de información para hacer una investigación crítica, fundamentada y propositiva con la finalidad de mejorar la práctica educativa, se puede decir que este propósito sí se logró en gran medida.

Lo anterior se fundamenta en que, a partir de esa experiencia, han logrado cambios significativos en diversos aspectos. Por ejemplo, son capaces dar un sustento teórico a sus ensayos y trabajos de investigación que les solicitan en las otras asignaturas; pueden incluir citas de diferentes autores para que no sólo sean sus ideas sino que tengan un soporte en estudios de especialistas en el tema del que estén hablando.

Así podemos afirmar con relación al desarrollo del pensamiento científico que hubo un avance en su formación. Esto fue reforzado con la asistencia a conferencias de especialistas 
en diferentes tópicos en educación, que les permitió darse cuenta que al ser profesores tienen la oportunidad de llevar a cabo investigación científica y que en la medida en que dominen estas habilidades investigativas será más fácil llevarla a cabo con la seriedad y formalidad que requiere cualquier estudio de este tipo.

Los alumnos llegaron a un nivel de reflexión que tuvo impacto en la formación que están recibiendo, lo constataron en la investigación de campo, mencionaron haber tenido una experiencia que les permitió aprendizajes significativos que facilitaría su recorrido en cada uno de los semestres subsecuentes, además de su formación continua, ya que algunos piensan continuar en la línea de desarrollar investigación educativa después de su formación inicial, misma que fue sometida a críticas llegando a concluir que en la escuela normal no se considera la importancia de la investigación en la docencia con la relevancia necesaria como futuros formadores.

\section{Reflexiones finales}

La formación de docentes ha tenido a través de su historia diferentes metodologías que son producto de diversos enfoques epistemológicos mismos que sustentan y son alimentados a su vez por las teorías sociológicas, psicológicas y pedagógicas entre otras.

Así, se pueden considerar el enfoque de formación docente tradicional, el del Profesor racional técnico, el Profesor como un profesional reflexivo y el Profesor como un investigador intelectual crítico.

El plan de estudios que opera actualmente en México en las escuelas normales, fundamentalmente está delineado por el tercero. En dicho plan y en la literatura especializada en este enfoque, se pueden apreciar diferentes declaraciones que permiten deducir que el docente no solamente debe pensar en su práctica antes, durante y después de ella, para poder actuar tomando decisiones; sino que también debe de aprender a articular la teoría que le permitan desarrollar la praxis docente; sin embargo, como hemos comentado y como ha sido documentado, los procesos de formación inicial han carecido de actividades propias que desarrollen esta habilidad o competencia.

El normalista usualmente egresa sin conocer literatura especializada, ni instituciones o sitios web que la ofrezcan, además de que suele existir una actitud de desdén o poco aprecio a la teoría para usarla en la práctica y, en consecuencia, existen pocas competencias de carácter investigativo lo cual va en detrimento de su formación, ya que en 
los estudios mencionados, la investigación de su práctica sería una de las pocas maneras por las cuales el docente puede verdaderamente transformar su desempeño.

En función de ello, nos propusimos realizar un programa de intervención que fomentara el habitus investigativo. No podemos decir que lo hemos logrado. Para eso haría falta un estudio longitudinal que no se ha realizado. Sin embargo, las actividades planificadas bajo el espíritu constructivista y, ante todo, la idea de compartir decisiones con los integrantes de este programa basándonos en algunos de los postulados de la pedagogía radical en la cual la negociación es fundamental, es cómo podemos considerar que hubo en el grupo una actitud de superación y mejora constante en todos los que participamos en esta acción. El habitus aquí comienza.

Si bien es cierto que se adquirieron conocimientos y destrezas, lo más importante en el desarrollo del habitus: la disposición duradera, comenzó a operarse en estos alumnos. Podemos decir, también, que esto se debió fundamentalmente a la democratización de decisiones en la formación de una comunidad de investigación y a que los alumnos adquirieron un aprendizaje significativo, pues lo pudieron aplicar a su contexto, y se percataron de la necesidad de desarrollar este habitus para su futuro desarrollo profesional.

\section{REFERENCIAS}

Ander-Egg Ezequiel. (2005). Debates y propuestas sobre la problemática educativa. Algunas reflexiones sobre los retos del futuro inmediato. Argentina: HomoSapiens.

Ausubel, David Paul. (1976). Psicología educativa. México: Trillas.

Bonnewitz, Patrice. (2003). La sociología de Pierre Bourdieu. Buenos Aires: Claves Perfiles.

Bourdieu, Pierre. (1990). Sociología y cultura. México: Grijalbo.

Carrera Gonzalo, María José. (2000). Evolucionar como profesor. Diálogo, formación e investigación. Madrid: Pomares.

Dewey, John. (1989). Cómo pensamos. Nueva exposición de la relación entre pensamiento reflexivo y proceso educativo. Barcelona: Paidós.

Díaz Barriga Arceo, Frida. (1993). Iniciación a la práctica docente. México: CONALEP.

Ferry, Giles. (1990). El trayecto de la formación docente. México: UNAM- Paidós. 
Giroux, Henry. (1990). Los profesores como intelectuales. México: Paidós.

Kemmis Stephen y Mctaggart, Robin. (1992). Cómo planificar la investigación acción. Barcelona: Laertes.

Larrosa, Jorge. (2007). La experiencia y sus lenguajes (Serie Encuentros y seminarios). Barcelona: Universidad de Barcelona.

Latorre, Antonio. (2003). La investigación-acción. Conocer y cambiar la práctica educativa. Barcelona: Ed.Graó.

Lipman, Matthew. (2001). Pensamiento complejo y educación. Madrid, España: Ediciones de la Torre.

Liston y Zeichner (1993). Formación del Profesorado y Condiciones Sociales de la Escolarización. Madrid: Morata.

Lozano Andrade, Inés. (2010). Uso y pertinencia de la teoría para la práctica y formación de docentes de escuela secundaria. En: Estudios sobre formación docente. México: SEP-DGENAM.

Lozano Andrade, Inés y Mercado Cruz, Eduardo. (2009). Cómo investigar la práctica docente. México: ENSM-ISCEEM.

Lozano Andrade, Inés. (2006). Normalistas vs. Universitarios o técnicos vs. Rudos. La formación y práctica del docente de escuelas secundarias desde sus representaciones sociales. México: Plaza y Valdés.

Manen, Max. (2003). Investigación educativa y experiencia vivida. Barcelona: Idea Books.

Messina, Graciela. (1999). Investigación en o acerca de formación docente: un estado del arte en los noventa. Revista Iberoamericana de educación, (19). Disponible en http://www.rieoei.org/oeivirt/rie19a04.PDF

Perrenoud, Phillipe. (2002). La práctica reflexiva en el oficio de profesor: profesionalización y razón pedagógica. Barcelona: Graó.

Piaget, Jean. (1964). Seis estudios de psicología. Barcelona: Labor.

Sandoval, Etelvina et.al. (2009). Desafíos y posibilidades en la formación de maestros. México: UPN, The University of New Mexico, SFU.

Schön, Donald. (1992). La formación de profesionales reflexivos. Barcelona: Paidós.

Secretaría de Educación Pública. (1999). Plan de estudios de licenciatura en educación secundaria. México: SEP. 
Stenhouse, Lawrence. (1987). La investigación como base de la enseñanza. Madrid: Ed. Morata

Teaching and Learning International Survey (TALIS). (2009). Estudio Internacional sobre Docencia y Aprendizaje. OCDE. Recuperado de http://www.oecd.org/document/14

Vigotsky, Lev Semiónovich. (1979). El desarrollo de los procesos psicológicos superiores. Barcelona: Grijalbo.

Zeichner, Kennetth. (1993). El maestro como profesional reflexivo. Revista de Pedagogía (220). Barcelona, Fontalba, 44-49. 